\section{Mudança nos parâmetros antropométricos: a influência de um programa de intervenção nutricional e exercício físico em mulheres adultas}

\author{
Change in anthropometric parameters: \\ the impact of a nutritional intervention \\ program and physical exercise on adult women
}

\author{
1 Escola de Nutrição, \\ Universidade Federal da \\ Bahia, Salvador, Brasil. \\ 2 Centro de Ciências Exatas \\ e Tecnológicas, Universidade \\ Federal do Recôncavo da \\ Bahia, Cruz das Almas, \\ Brasil. \\ Correspondência \\ P. R. F. Costa \\ Escola de Nutrição, \\ Universidade Federal da \\ Bahia. \\ Rua Araujo Pinho 32 \\ Salvador, $B A$ \\ 40110-150, Brasil. \\ priscilarf@yahoo.com.br
}

\begin{abstract}
Obesity prevalence is increasing worldwide, with inadequate nutrition and physical inactivity as the main risk factors. The objective was to evaluate the impact of a nutritional intervention and physical activity program on body mass index (BMI) and waist circumference (WC) in adult women. This quasi-experimental study, lasting 12 months, enrolled 69 adult women that reported physical activity three times a week, besides receiving nutritional counseling and lectures on healthy living, evaluated at baseline and at 6 and 12 months of follow-up. Lower levels of physical activity and higher intake of high-risk foods were associated with a $2.28 \mathrm{~cm}$ increase in mean WC, has compared to higher physical activity and lower intake of high-risk foods $(p<0.01)$. Lower levels of physical activity associated with high intake of protective foods increased the mean BMI by $0.68 \mathrm{~kg} / \mathrm{m}^{2}$, as compared to higher physical activity and high intake of protective foods. The intervention was positively associated with the target parameters.
\end{abstract}

Obesity; Anthropometry; Exercise; Women's Health

\author{
Priscila Ribas de Farias Costa 1 \\ Ana Marlúcia Oliveira Assis ${ }^{1}$ \\ Maria da Conceição Monteiro da Silva 1 \\ Mônica Leila Portela de Santana 1 \\ Jacqueline Costa Dias 1 \\ Sandra Maria Conceição Pinheiro 2 \\ Nedja Silva Santos 1
}

\section{Introdução}

O excesso de peso destaca-se como uma das doenças de mais elevada prevalência em todo o mundo. Em 2002, estimativas da Organização Mundial da Saúde (OMS) 1 indicavam a existência de mais de 1 bilhão de adultos portadores dessa morbidade, sendo mais de 300 milhões obesos. A cada ano, aproximadamente 2,6 milhões de pessoas morrem em conseqüência do excesso de peso ${ }^{2}$. Essa enfermidade se constitui também em importante fator de risco para outras doenças crônicas como diabetes tipo II, hipertensão arterial, cardiopatias e certos tipos de câncer, aumentando o risco cardiovascular 3 .

Na América Latina, a ocorrência da obesidade vem crescendo, especialmente entre mulheres adultas com baixa escolaridade 4. Para o Brasil, dados de 2003 da Pesquisa de Orçamento Familiar (POF) 5 revelaram que o excesso de peso é maior entre as mulheres, afetando $40 \%$ delas e, destas, 13,1\% são obesas. Ressalta-se que o crescimento da prevalência da obesidade em mulheres ocorre em sua maioria na classe de menor rendimento ${ }^{6}$. Estudos avaliando a tendência da obesidade no Brasil mostraram haver uma redução na prevalência dessa morbidade entre mulheres com renda elevada e aumento entre aquelas com nível de renda mais baixo 7,8.

$O$ excesso de peso tem etiologia multifatorial e complexa, incluindo fatores de risco modificáveis como a inatividade física e a alimentação 
inadequada 1. Entre as práticas alimentares associadas com o excesso de peso tem-se o consumo de alimentos com elevada densidade calórica, com alta concentração de carboidratos simples, gordura total, ácidos graxos saturados e trans saturados, bem como a baixa ingestão de frutas e hortaliças 1,9. Esse tipo de dieta, associada ao sedentarismo, são identificados como os principais fatores etiológicos da obesidade no mundo 10 .

Considerando a inatividade física, dados da OMS 11 revelam que $17 \%$ da população mundial é sedentária e $60 \%$ não atinge o mínimo de atividade física recomendado para ser considerado ativo. Para o Brasil, um estudo realizado com mulheres no Sul do país identificou prevalência de sedentarismo de 37\%. Além disto, 59,3\% relataram praticar atividade física menos de três vezes na semana ${ }^{12}$. Dados de um estudo realizado nas regiões Sul e Nordeste do Brasil indicaram prevalências de $31,8 \%$ e $58 \%$ de sedentarismo, respectivamente 13 .

A prática de exercíciosfísicos tem sido ressaltada como fator de prevenção primária e de suporte terapêutico de diversas enfermidades crônicas 14 . As evidências são consistentes para definir como positiva a influência da atividade física aeróbica na prevenção e no controle da obesidade 15 .

Assim, a OMS lançou a Estratégia Global em Alimentação Saudável, Atividade Física e Saúde (EG/OMS) 1, considerando que a alimentação e o estilo de vida saudáveis constituem estratégias capazes de diminuir a ocorrência e a gravidade das doenças crônicas não transmissíveis, estratégia adotada também pelo Brasil, implementadas pelo Ministério da Saúde, na tentativa de reduzir a carga das doenças crônicas não transmissíveis 16. Diante disso, o objetivo deste estudo foi avaliar a influência das recomendações da EG/OMS sobre o estado antropométrico de mulheres adultas.

\section{Métodos}

\section{Desenho e amostra do estudo}

Trata-se de um estudo de intervenção quasi-experimental, do tipo antes e depois 17, com duração de 12 meses, realizado no Município de Mutuípe, Bahia, Brasil, no período de março de 2006 a março de 2007. A amostra foi construída com base na adesão voluntária de mulheres que participavam do projeto Dieta, Exercício Físico e Doenças Crônicas Não Transmissíveis - Um Estudo de Intervenção, que incluía 120 pessoas de 20 a 59 anos de ambos os sexos.

Adotaram-se como critérios a autorização médica para a prática do exercício físico, baseada na avaliação clínica (incluindo o exame de eletrocardiograma e da capacidade e resistência física) e a disposição dos indivíduos de freqüentar regularmente as sessões de exercício físico e de aderir ao atendimento nutricional. Assim, das 120 pessoas que participavam do programa original, 69 atenderam a esses critérios e concordaram em participar do estudo. Esta amostra tem poder de 95,3\% para detectar uma redução de $10 \%$ na média do índice de massa corporal (IMC), considerando a média de $23,15 \mathrm{~kg} / \mathrm{m}^{2} \pm 4,0518$; e poder de $98,6 \%$ de detectar redução de $10 \%$ na média da circunferência da cintura (CC), considerando a média de $80,8 \mathrm{~cm} \pm 12,319$.

\section{Critérios de exclusão}

Foram excluídos do estudo indivíduos com parecer médico e de aptidão física desfavoráveis, estado de gestação e lactação, além de estado de deficiência física que impossibilitasse a prática de exercícios físicos. Devido ao baixo número de homens participantes do estudo original, optouse por incluir somente as mulheres na subamostra desse estudo.

\section{A intervenção}

A intervenção foi baseada nas recomendações da EG/OMS 1, de acordo com os seguintes pressupostos:

a) Alimentação saudável: foi construída pela orientação com vistas à limitação da ingestão energética procedente das gorduras; a substituição do consumo de gorduras saturadas pelas insaturadas; a exclusão das gorduras trans da dieta; o aumento do consumo de frutas e verduras para um mínimo diário de $400 \mathrm{~g}$, aumento do consumo de oleaginosas e de alimentos com elevado teor de fibras; e a limitação do consumo de açúcares livres e sal.

b) Exercício físico: adotou-se um protocolo com a seguinte logística - o exercício aeróbio praticado em quadra coberta, com duração de 60 minutos, caracterizado como de intensidade leve a moderada, incluindo o aquecimento, com duração de 10 minutos; 40 minutos de ginástica aeróbica e 10 minutos de resfriamento, realizado três vezes na semana, com duração de no mínimo 30 minutos. Essa atividade foi realizada sob a orientação de um professor de educação física e ocorria no período da manhã (das 6 as 7 horas). Nos demais dias da semana as participantes eram orientadas a realizarem caminhadas.

A intervenção durou 12 meses e sua logística foi norteada pela seguinte organização metodológica: 
a) Sessões de orientação nutricional, de caráter individual e periodicidade de seis meses, totalizando três sessões durante os 12 meses do seguimento. As orientações eram realizadas individualmente por nutricionistas treinados, de maneira oral e escrita, baseadas na EG/OMS 1. Em cada sessão eram também realizadas as coletas de sangue para dosagem dos parâmetros de interesse, a avaliação antropométrica e do consumo alimentar. Na primeira sessão individual foram coletados os dados referentes ao estilo de vida, demográficos, sociais e econômicos.

b) Realização de sessões em grupo de exercício físico aeróbico, três vezes na semana, com duração de uma hora, sob a orientação de um professor de educação física, durante 12 meses. Nos demais dias, os indivíduos eram orientados a realizarem caminhadas de pelo menos 30 minutos. c) Realização de palestras a cada dois meses, com temas referentes à alimentação, nutrição e saúde, enfatizando a relação entre adoção de hábitos alimentares e estilo de vida saudáveis e a melhoria da qualidade de vida.

d) A cada mês eram transmitidas entrevistas sobre alimentação e hábitos de vida saudáveis pela rádio local, abertas a questionamentos dos ouvintes por telefone.

e) Em cada contato individual (sessões de atendimento nutricional) era monitorada a adesão ao protocolo do estudo.

\section{Procedimentos éticos}

O estudo foi apreciado e aprovado pelo Comitê de Ética da Escola de Nutrição da Universidade Federal da Bahia, conforme determina a Resolução $n^{\circ}$. 196/96 do Conselho Nacional de Saúde. Os pacientes elegíveis foram informados dos objetivos do estudo e aqueles que concordaram com os seus termos foram convidados a assinar o termo de consentimento livre e esclarecido.

Todos os indivíduos que apresentaram problemas de saúde já avaliados ou não pelo médico foram encaminhados ao serviço de saúde local. O mesmo procedimento foi adotado com aquele que apresentou problemas de saúde durante o seguimento.

\section{Coleta de dados e definição de variáveis}

Informações demográficas, sócio-econômicas e de estilo de vida

As informações demográficas, sócio-econômicas e do estilo de vida foram coletadas por meio de questionário respondido pelos investigados ao início do seguimento.
Todas as informações foram coletadas por equipe de pesquisadores treinados e devidamente capacitados. Os procedimentos de coleta dos dados foram padronizados, como medida de controle da qualidade e consistência das informações.

\section{Medidas antropométricas}

\section{- Peso, altura e circunferência da cintura}

Para a obtenção do peso foi utilizada balança digital portátil Filizola (São Paulo, Brasil), com capacidade para $150 \mathrm{~kg}$ e precisão de $100 \mathrm{~g}$. O procedimento de pesagem foi realizado em duplicata e a média entre as duas medidas foi adotada como medida final. $\mathrm{O}$ erro aceitável foi de $0,1 \mathrm{~kg}$. A altura foi aferida por meio de estadiômetro marca Leicester Height Measure (SECA; Hamburgo, Alemanha), com a leitura realizada no milímetro mais próximo. $\mathrm{O}$ indivíduo foi pesado e medido de acordo com as técnicas de Lohman et al. 20. A CC foi medida em duplicata, com uso da fita métrica inelástica, de fibra de vidro, com escala em centímetros. O erro aceitável entre as duas medições foi de $0,1 \mathrm{~cm}$. A técnica utilizada foi a proposta pela OMS 21 .

\section{Indicadores antropométricos}

\section{- Índice de massa corporal (IMC) e a circunferência da cintura (CC)}

O IMC 22 e a CC foram usados na forma contínua, como variável resposta. Para o IMC foram considerados os pontos de corte da OMS 21 para peso normal $\left(18,5 \mathrm{~kg} / \mathrm{m}^{2}-24,9 \mathrm{~kg} / \mathrm{m}^{2}\right)$ e excesso de peso (sobrepeso e obesidade) $\left(\geq 25 \mathrm{~kg} / \mathrm{m}^{2}\right)$. Para a CC adotaram-se $<80 \mathrm{~cm}$ para a normalidade (adequada) e valor maior do que $80 \mathrm{~cm}$ para a CC elevada 21 . O uso destes pontos de corte ocorreu somente na descrição do estado antropométrico da população.

\section{Consumo alimentar}

As informações referentes ao consumo alimentar foram coletadas ao baseline e a cada seis meses, utilizando-se da técnica da freqüência do consumo alimentar, composta por 94 alimentos e com oito possibilidades de resposta de consumo: diário; 1 vez na semana; 2 vezes na semana; 3 vezes na semana; 4 vezes na semana; 5 vezes na semana; 6 vezes na semana e raramente/nunca.

Essas informações foram tratadas de acordo com a metodologia proposta por Monteiro et al. 23. Nessa metodologia, o cômputo geral da fre-qüência de consumo é convertido em es- 
cores, multiplicando todas as freqüências semanais por 4 (número de semanas no mês) e dividindo-as por 30 (número de dias no mês), encontrando-se, assim, o escore médio de consumo diário para cada alimento no período de um mês. Os escores variavam de 1 (consumo diário) a 0 (opção de consumo "raramente/nunca"). Um consumo de 5 vezes na semana, por exemplo, correspondeu a um escore de 0,67 , obtido pela seguinte equação: consumo de 5 vezes na semana multiplicado por 4 semanas e dividido por 30 dias.

O consumo diário por grupo de alimentos tomou a seguinte caracterização: escore médio de consumo diário entre 0,0 e 0,32 (consumo diário baixo), entre 0,33 e 0,65 (consumo diário médio) e entre 0,66 e 1,0 (consumo diário elevado).

Estabelecidos os escores, foram construídos dois grupos para caracterizar o consumo alimentar, baseando-se na metodologia utilizada por outro autor 23 , sendo adaptada às características da população e região em estudo.

Assim, foram construídos dois grupos de consumo alimentar, com a seguinte caracterização: a) Grupo de risco: formado por alimentos considerados de risco para doenças crônicas não transmissíveis, ricos em gorduras saturadas, cloreto de sódio e açúcares simples. Para ser suportado pelo modelo estatístico, a categoria de referência foi representada pelo escore de consumo diário baixo e o risco foi interpretado como o escore de consumo diário médio/elevado. b) Grupo proteção: foi formado por alimentos protetores para doenças crônicas não transmissíveis, ricos em fibras, vitaminas, minerais e com baixa densidade calórica 24 . Para este grupo de alimentos, a categoria de referência foi representada pelo escore de consumo diário elevado e a categoria de risco foi o escore de consumo diário baixo/médio.

\section{$\underline{\text { Nível de atividade física }}$}

Para identificar o nível de atividade física, foi utilizada a forma simplificada da versão 8 do Questionário Internacional de Atividade Física (IPAQ), validado em uma amostra da população brasileira ${ }^{25}$. As informações dizem respeito ao nível de atividade do indivíduo nos últimos sete dias de cada período do seguimento: ao início, aos 6 meses e aos 12 meses. Esse instrumento, além da prática de exercício físico, considera também as atividades realizadas no domicílio, lazer e para deslocamento. A avaliação foi realizada utilizando-se o consenso preconizado pelo Centro de Estudos do Laboratório de Atividade Física de São Caetano do Sul (CELAFISCS) e o Centers for Disease Control ad Prevention
(CDC), considerando os critérios de freqüência e duração, que classificam as pessoas em: muito ativo, ativo, irregularmente ativo e sedentário ${ }^{26}$. Para este estudo, as participantes foram classificadas como: muito ativas/ativas e irregularmente ativas/sedentárias.

O questionário IPAQ tem a vantagem de ter sido validado para diferentes populações, possibilitando comparações com outros países. Porém, possui o inconveniente de não ser recomendável quando a amostra é pequena. Entretanto, diante das poucas alternativas acessíveis para realizar tal procedimento, este questionário tem sido amplamente empregado, inclusive em pequenas amostras 27,28 .

\section{Índice de Estilo de Vida}

Com o intuito de avaliar a influência da alimentação saudável e do exercício físico, conjuntamente sobre os parâmetros de interesse, foi criado um Índice de Estilo de Vida a partir das variáveis independentes principais do estudo.

O consumo alimentar foi representado pelo escore diário médio de freqüência de consumo (com base na classificação de grupos de risco e de proteção) e o exercício físico foi representado pelo nível de atividade no qual a participante se enquadrava (muito ativa/ativa e irregularmente ativa/sedentária). Para compor o índice, essas variáveis foram convertidas em variáveis dicotômicas e usadas no modelo estatístico como variantes no tempo.

Assim, foram criadas quatro categorias possíveis para cada Índice de Estilo de Vida, levando em consideração os grupos de consumo alimentar e o nível da atividade física. Para o grupo de alimentos de risco, essa classificação tomou a seguinte conotação:

a) Consumo baixo de alimentos do grupo de risco para doenças crônicas não transmissíveis + nível elevado de atividade física (grupo referência).

b) Consumo médio/elevado de alimentos do grupo de risco para doenças crônicas não transmissíveis + nível elevado de atividade física (categoria 1).

c) Consumo baixo de alimentos do grupo de risco para doenças crônicas não transmissíveis + nível baixo de atividade física (categoria 2).

d) Consumo médio/elevado de alimentos do grupo de risco para doenças crônicas não transmissíveis + nível baixo de atividade física (categoria 3).

Já para o grupo protetor, a categorização tomou a seguinte conotação:

a) Consumo elevado de alimentos do grupo protetor + nível elevado de atividade física (grupo referência). 
b) Consumo elevado de alimentos do grupo protetor + nível baixo de atividade física (categoria 1).

c) Consumo baixo de alimentos do grupo protetor + nível elevado de atividade física (categoria 2).

d) Consumo baixo de alimentos do grupo protetor + nível baixo de atividade física (categoria 3).

\section{Exames laboratoriais}

A coleta do sangue ocorreu no início, aos 6 e aos 12 meses do seguimento, sendo realizada pela manhã por técnico de laboratório treinado e sob a supervisão de bioquímico, observando-se o jejum de pelo menos 12 horas e abstinência ao álcool de 72 horas. Foram coletados $15 \mathrm{~mL}$ de sangue, por meio de tubos de vacutainer (BD; Franklin Lakes, Estados Unidos) estéreis e descartáveis. Uma quantidade de $10 \mathrm{~mL}$ do sangue foi acondicionada em tubos sem anticoagulante para obtenção do soro. O sangue permaneceu em descanso por cerca de 30 minutos à temperatura ambiente e centrifugado a 3000rpm por 5 minutos para separação do soro, que foi utilizado na determinação dos triglicerídeos. As amostras hemolisadas foram excluídas. As dosagens dos triglicérides foram realizadas no laboratório referência do município. Os $5 \mathrm{~mL}$ restantes foram acondicionados e congelados para dosagens de outros princípios de interesse.

Neste estudo utilizou-se triglicérides como co-variável pela conhecida relação com o peso corpóreo, em especial com o IMC, uma vez que o excesso de peso está associado ao aumento dos valores de triglicérides séricos, podendo funcionar como uma variável de confusão 29.

\section{Sazonalidade}

Considerando a influência que a sazonalidade exerce sobre o estado antropométrico do indivíduo, optou-se por incluir no desenho do estudo uma variável que pudesse exercer o papel de ajuste na análise estatística. Esta variável foi construída levando em consideração a época do seguimento em que foram realizadas as medições antropométricas, tomando como parâmetro os 12 meses de seguimento do estudo ${ }^{30}$. Ela integrou o modelo como co-variável, na forma contínua e variante no tempo.

\section{Processamento dos dados}

Após as rotinas de controle das informações coletadas, os dados foram digitados, à medida que chegavam de campo. Dois digitadores foram designados para este fim. Foi utilizado o programa Epi Info (CDC, Atlanta, Estados Unidos), que tem caráter simplificado e evita erros grosseiros na entrada de dados. Todos os dados foram redigitados por outro digitador, para efeito de análise da consistência.

\section{Análise dos dados}

As variáveis sócio-econômicas e do estilo de vida das participantes foram categorizadas para integrarem os modelos estatísticos, e encontram-se de maneira auto-explicativa nas tabelas.

Para a análise estatística descritiva foi utilizada a prevalência, com vistas à descrição da população do estudo. Para comparar a mudança nos valores médios dos parâmetros antropométricos e de consumo alimentar, ao inicio e ao final do seguimento, foi utilizado o teste $t$ pareado, para amostras dependentes e com distribuição normal.

Para avaliar a relação das variáveis respostas e aquela de exposição, ao longo do tempo, utilizou-se a técnica equação de estimação generalizada (GEE), apropriada para respostas contínuas e medidas repetidas, pois reflete a relação longitudinal entre as variáveis respostas e as variáveis preditoras correspondentes, considerando a correlação entre as medidas em cada momento no tempo ${ }^{31}$. Assim, foram incorporadas na GEE as medidas repetidas realizadas no seguimento (ao baseline, aos 6 e aos 12 meses). Foi utilizada a técnica backward, que inclui a variável uma a uma no modelo, sendo eliminadas aquelas que não atingiam o valor de p previamente estipulado. Para a permanência no modelo, foi adotado como critério o nível de significância menor do que $5 \%$. Foram adotados os resultados provenientes do modelo robusto, que incorpora na sua matriz de correlação as variações na coleção de dados.

As variáveis respostas foram representadas pela mudança na CC e IMC do indivíduo ao longo do seguimento e inserido no modelo na forma contínua. Foi construído um modelo de GEE para cada uma dessas variáveis.

Adotou-se o Índice de Estilo de Vida como variável de exposição principal, para avaliar a influência conjunta da alimentação saudável e do exercício físico sobre os parâmetros de interesse. Essa variável foi utilizada de forma categorizada (0 referência e 1 risco) e variante no tempo. As demais variáveis integraram o modelo como covariáveis ou variáveis de ajuste.

Foram explorados possíveis variáveis de confusão e termos de interação, utilizando-se do referencial teórico disponível na literatura e do próprio conjunto de dados deste estudo ${ }^{32}$. Foi utilizado o pacote estatístico SAS for Windows versão 8.0 (SAS Inst., Cary, Estados Unidos) para a análise dos dados. 


\section{Resultados}

Houve perda de $8,7 \%(n=6)$ no seguimento. A análise estatística indicou não haver diferença nas variáveis sócio-econômicas, de estilo de vida e variáveis resposta entre o grupo de participantes que permaneceu no estudo e aquele que foi perdido ao longo do seguimento (dados não apresentados).

As características sócio-demográficas e a distribuição percentual dos indicadores antropométricos das mulheres estão apresentadas na Tabela 1. Observou-se predominância da idade maior de 35 anos; o nível de renda mostrou-se semelhante para as três categorias; o nível de es-

Tabela 1

Características sócio-demográficas, de estilo de vida e antropométricas das participantes do estudo no baseline. Mutuípe, Bahia, Brasil, 2006 ( $N=69$ ).

\begin{tabular}{|c|c|c|}
\hline Variáveis & n & $\%$ \\
\hline \multicolumn{3}{|l|}{ Idade (anos) } \\
\hline$<35$ & 12 & 17,4 \\
\hline$\geq 35$ & 57 & 82,6 \\
\hline \multicolumn{3}{|l|}{ Renda (salários mínimos) } \\
\hline$\leq 1$ & 25 & 36,2 \\
\hline Até 2 & 21 & 30,4 \\
\hline$>2$ & 23 & 33,3 \\
\hline \multicolumn{3}{|l|}{ Escolaridade } \\
\hline Analfabeto & 8 & 11,6 \\
\hline Elementar ou fundamental completo/incompleto & 27 & 39,1 \\
\hline Médio ou superior completo/incompleto & 34 & 49,3 \\
\hline \multicolumn{3}{|l|}{ Situação conjugal } \\
\hline Casado & 45 & 65,2 \\
\hline Outros (solteiro/divorciado/viúvo) & 24 & 34,8 \\
\hline \multicolumn{3}{|l|}{ Ocupação } \\
\hline Empregado & 33 & 47,8 \\
\hline Desempregado & 36 & 52,2 \\
\hline \multicolumn{3}{|l|}{ Tabagismo } \\
\hline Fumante/Ex-fumante & 29 & 42,0 \\
\hline Não fumante & 40 & 58,0 \\
\hline \multicolumn{3}{|l|}{ Consumo de bebida alcoólica } \\
\hline Sim & 42 & 60,9 \\
\hline Não & 27 & 39,1 \\
\hline \multicolumn{3}{|l|}{ Nível de atividade física } \\
\hline Muito ativo/Ativo & 21 & 30,4 \\
\hline Irregularmente ativo/Sedentário & 48 & 69,6 \\
\hline \multicolumn{3}{|l|}{ Índice de massa corporal } \\
\hline Eutrofia & 10 & 14,5 \\
\hline Sobrepeso/Obesidade & 59 & 85,5 \\
\hline \multicolumn{3}{|l|}{ Circunferência de cintura } \\
\hline Adequada & 25 & 36,2 \\
\hline Elevada & 44 & 63,8 \\
\hline
\end{tabular}

colaridade elementar ou fundamental e o analfabetismo juntos são ligeiramente superiores ao observado para o nível de escolaridade médio ou superior completo ou incompleto. No inicio do estudo a maioria se declarou não fumante e o consumo de algum tipo de bebida alcoólica foi referido por $60,9 \%$ das participantes. No início do estudo quase $70 \%$ das mulheres eram irregularmente ativas ou sedentárias.

A distribuição percentual dos indicadores antropométricos indica prevalência de $85,5 \%$ de excesso de peso, segundo o IMC. Para a CC, valores considerados elevados $(>80 \mathrm{~cm})$ foram encontrados em quase $64 \%$ do grupo (Tabela 1 ).

A diferença de média, observada ao final do seguimento para os parâmetros avaliados, está apresentada na Tabela 2 . Os resultados do teste $t$ pareado indicaram declínio estatisticamente significante na média da CC ( $\mathrm{p}=0,015)$. Para o IMC, não foi observada diferença estatisticamente significante entre as médias $(\mathrm{p}=0,987)$.

Ao avaliar o escore médio de consumo diário por grupos de alimentos, ao inicio do estudo, identificou-se maior ingestão de gorduras e frituras (escore $=0,71$ ) e mais baixo consumo de frutas e hortaliças (escore $=0,55$ ) e alimentos magros de origem animal (escore $=0,55$ ). No padrão de consumo de frutas e hortaliças, identificouse aumento de 0,1 no escore médio de consumo diário após a intervenção (alterando de 0,55 ao início do estudo para 0,65 escore ao final do seguimento; $\mathrm{p}<0,01$ ) (dados não apresentados).

Neste estudo, registrou-se aumento no nível de atividade física na categoria muito ativas/ativas ao final do seguimento (73,9\%), sendo essa diferença estatisticamente significante quando comparada à proporção inicial $(30,4 \%)(\mathrm{p}<0,01)$ (dados não apresentados).

Foram realizados dois modelos de análise de GEE, um para cada um dos parâmetros antropométricos avaliados (Tabela 3). A média do IMC na população ao longo do período de seguimento foi $27,19 \mathrm{~kg} / \mathrm{m}^{2}(\mathrm{p}<0,01)$. Os resultados indicaram que as mulheres com menores níveis de atividade física e consumo elevado de alimentos protetores apresentaram incremento de $0,68 \mathrm{~kg} /$ $\mathrm{m}^{2}$ na média do IMC $(\mathrm{p}=0,035)$ nos 12 meses do seguimento, quando comparadas àquelas com maiores níveis de atividade física e consumo elevado de alimentos protetores. Esses resultados foram controlados pela sazonalidade, renda, idade e história familiar de obesidade.

Os resultados da análise de GEE indicou que a média da CC na população ao longo do período de seguimento foi de $87,95 \mathrm{~cm}(\mathrm{p}<0,01)$. Os resultados mostraram que mulheres com menores níveis de atividade física e elevado consumo de alimentos de risco para doenças crônicas não 
Diferença de média das variáveis respostas antes e após a intervenção. Mutuípe, Bahia, Brasil, 2006.

\begin{tabular}{lcccc}
\hline Variáveis & Média no início & Média no final & Diferença de média & Valor de $\mathbf{p}$ * \\
\hline Índice de massa corporal $\left(\mathrm{kg} / \mathrm{m}^{2}\right)$ & 28,77 & 28,76 & $-0,003$ & 0,987 \\
Circunferência de cintura $(\mathrm{cm})$ & 92,50 & 91,10 & $-1,240$ & 0,015 \\
\hline
\end{tabular}

* Teste t pareado.

Modelos de equação de estimação generalizada (GEE) para a circunferência da cintura e o índice de massa corporal. Mutuípe, Bahia, Brasil, 2006.

\begin{tabular}{|c|c|c|c|}
\hline Variáveis & $\beta$ & Erro-padrão & Valor de $\mathrm{p}$ \\
\hline \multicolumn{4}{|l|}{ Circunferência de cintura } \\
\hline Intercepto * & 88,01 & 1,975 & $<0,01$ \\
\hline Maior nível de atividade física e baixo consumo de alimentos de risco & - & - & - \\
\hline Menor nível de atividade física e elevado consumo de alimentos de risco & 2,23 & 0,862 & $<0,01$ \\
\hline Menor nível de atividade física e baixo consumo de alimentos de risco & $-0,16$ & 1,118 & 0,886 \\
\hline Maior nível de atividade física e elevado consumo de alimentos de risco & 1,37 & 1,099 & 0,211 \\
\hline \multicolumn{4}{|l|}{ Índice de massa corporal } \\
\hline Intercepto ** & 26,65 & 0,622 & $<0,01$ \\
\hline Maior nível de atividade física e elevado consumo de alimentos protetores & - & - & - \\
\hline Menor nível de atividade física e baixo consumo de alimentos protetores & 0,15 & 0,263 & 0,144 \\
\hline Menor nível de atividade física e elevado consumo de alimentos protetores & 0,68 & 0,312 & 0,035 \\
\hline Maior nível de atividade física e baixo consumo de alimentos protetores & 0,38 & 0,202 & 0,457 \\
\hline
\end{tabular}

* Ajustado pela sazonalidade, história familiar de obesidade e triglicérides séricos;

** Ajustado pela sazonalidade e história familiar de obesidade.

transmissíveis tiveram um aumento de $2,28 \mathrm{~cm}$ na média da CC, durante o período do seguimento, quando comparadas àquelas com maiores níveis de atividade física e, baixo consumo de alimentos de risco para as doenças crônicas não transmissíveis $(\mathrm{p}<0,01)$, sendo esse resultado controlado pela sazonalidade, renda, idade, história familiar de obesidade e níveis de triglicérides séricos.

Foram testados possíveis termos de interação. Entretanto, estes não apresentaram significância estatística $(\mathrm{p}<0,05)$.

\section{Discussão}

No presente estudo registrou-se perda amostral que pode ser considerada baixa para uma intervenção de 12 meses, se comparada a estudos com metodologia similar 23,33. Essas perdas ocorreram em sua maioria por migração para outros municípios. Mas as perdas não introduziram alteração na composição dos grupos de participação em qualquer período do seguimento, nas características antropométricas e biológicas das participantes.

Os resultados indicam que o menor nível de atividade física e o elevado consumo diário de alimentos de risco para doenças crônicas não transmissíveis podem explicar o aumento da CC dessas mulheres. Indica também que o menor nível de atividade física, ainda que o consumo de alimentos protetores seja elevado, isoladamente não assegura a redução dos valores do IMC. Esses resultados podem ser interpretados como a indicação necessária da associação da prática do exercício físico regular com o consumo de alimentos protetores, a exemplo do preconizado pela EG/OMS 1. Vale ressaltar ainda que a recomendação tradicional de no mínimo 150 minutos semanais de atividade física de intensidade leve a moderada - como a preconizada no presente estudo -, que tem como base principalmente os 
efeitos positivos da atividade física sobre a doença cardiovascular e sobre o diabetes mellitus, pode não constituir o tempo e a duração adequados para fomentar a perda de peso ${ }^{34}$, o que pode ter levado à baixa influência do exercício físico sobre os parâmetros antropométricos avaliados.

Esses resultados corroboram achados de estudos que mostram que mudanças positivas no consumo alimentar e no nível de atividade física podem controlar o excesso de peso, sendo importante para a prevenção e tratamento dessa morbidade 1. Esses resultados são registrados tanto por intervenções controladas 33,35 quanto por estudos transversais 36,37. A associação da intervenção nutricional com o exercício físico tem apresentado melhores benefícios na redução do peso corpóreo de mulheres obesas do que a intervenção nutricional isoladamente 23 . Entretanto, há registro do benefício desta intervenção por seis meses adotada de forma isolada em pacientes diabéticos, embora os autores argumentem que os efeitos podem ser intensificados quando tais intervenções ocorrem conjuntamente 38 .

É interessante comentar que neste estudo a intervenção exerceu influências diferentes sobre os indicadores antropométricos das participantes, com redução significante nos valores médios da CC, sem influenciar o IMC. Compreende-se que a CC reflete a distribuição corporal de gordura e pareceu ser mais sensível às mudanças promovidas pela atividade física e alteração no padrão do consumo alimentar, sendo rapidamente afetada pela perda de peso ${ }^{39}$. Por outro lado, o IMC reflete a mudança ocorrida na composição corporal de indivíduos submetidos a exercícios físicos, como o aumento na oxidação de gorduras, promovendo a perda gradual desta substância com conseqüente elevação da massa magra, que possui peso líquido maior que o da gordura, o que pode ser interpretado erroneamente como excessivo ganho de peso 40.

É incontestável que o baixo nível de atividade física das mulheres no início do seguimento se assemelha ao padrão de sedentarismo apresentado pela população brasileira ${ }^{41}$. No presente estudo, houve aumento no nível de atividade física após um ano de estímulo à prática de exercício físico em grupo, embora uma parcela não tenha conseguido participação freqüente no programa. Isso reforça a conhecida dificuldade de adesão ao exercício físico na população geral 26.

O padrão alimentar apresentado pelas mulheres no início do estudo, caracterizado por elevado consumo de gorduras e frituras e baixa ingestão de frutas e hortaliças, parece representar o hábito alimentar dos brasileiros, cuja transformação observada nas últimas décadas indicou redução no consumo de cereais, leguminosas, raízes e tubérculos e aumento no consumo de gorduras, além do consumo insuficiente de frutas e hortaliças ${ }^{42}$. Essas mudanças podem explicar o aumento da prevalência de obesidade no país. Neste período, o excesso de peso aumentou de $24 \%$ (em 1975) para $40 \%$ (em 2003) 16. Estas transformações seguramente atingiram também a população de pequenos municípios, a exemplo do município baiano onde este estudo foi desenvolvido.

Entende-se que esta intervenção pode alterar de forma positiva o panorama epidemiológico dessas doenças entre as participantes, sendo assim capaz de promover mudanças positivas no estilo de vida após um ano de intervenção, com aumento nos níveis de atividade física e no consumo diário de frutas e hortaliças. Para modificar a estrutura da dieta de um indivíduo são necessários programas de aconselhamento nutricional gradativo e em longo prazo, sendo as mulheres o grupo chave, uma vez que geralmente elas são responsáveis por controlar as práticas alimentares da família 43 .

Os resultados deste estudo são relevantes do ponto de vista epidemiológico e podem representar uma adequada estratégia para redução da CC de mulheres adultas e sem doenças crônicas instaladas, atuando como mecanismo preventivo das doenças crônicas não transmissíveis. Em grupos de risco, que já apresentam tais morbidades, é provável que o efeito de intervenções deste tipo sobre o estado de saúde do indivíduo apresente expressão ainda maior.

Entretanto, cabe aqui discutir brevemente as vantagens e desvantagens do desenho de estudo quasi-experimental do tipo antes e depois, utilizado para esta intervenção, apesar do cuidado em seu planejamento, operacionalização e análise.

A ausência de um grupo controle não permite afirmar que os resultados encontrados nesse estudo foram realmente devido à intervenção, uma vez que o seu desenho não se enquadra entre aqueles aleatorizados controlados. Estes são os estudos com maior credibilidade para avaliar causalidade, especialmente devido à aleatorização dos grupos, que distribui eqüitativamente as variáveis confundidoras entre os grupos controle e intervenção. Entretanto, existem situações de ordem ética ou logística em que este tipo de estudo não é aconselhável. Uma das mais freqüentes, e que se aplica ao presente estudo, é a consideração ética. Quando a eficácia de uma intervenção é bem conhecida e estabelecida, comprovada por estudos anteriores, então a questão ética surge, não permitindo a negação do tratamento a um grupo de indivíduos 44,45 . 
Existem métodos que tornam mais convincentes os resultados de um estudo quasi-experimental, como o rigor metodológico e técnicas analíticas, visando controle de potenciais confundidores. Além disso, aumentar o número de medida após a intervenção, como foi realizado no presente estudo, fornece evidências contra o confundimento e explicações alternativas para as associações observadas. Ressalta-se também que revisões de estudos experimentais e quasiexperimentais têm demonstrado que os achados obtidos nesses estudos são similares 45 .

Este estudo soma-se àqueles que adotam estratégias eficazes e viáveis em Saúde Pública, que podem ser desenvolvidas no âmbito dos serviços de saúde. E coloca mais uma vez em realce que a redução do consumo de alimentos considerados de risco para doenças crônicas

\section{Resumo}

A obesidade apresenta ocorrência crescente em todo o mundo, sendo alimentação inadequada e sedentarismo seus importantes fatores de risco. O objetivo foi avaliar a influência de um programa de intervenção nutricional e exercício físico sobre o índice de massa corporal (IMC) e circunferência da cintura (CC) de mulheres adultas. Trata-se de um estudo quasi-experimental, duração de 12 meses, participando 69 mulheres adultas, submetidas à atividade física três vezes na semana, orientação nutricional e palestras sobre vida saudável, avaliadas ao início, 6 e 12 meses do seguimento. Menores níveis de atividade física e alto consumo de alimentos de risco elevaram em $2,28 \mathrm{~cm}$ a média da CC das mulheres, quando comparado àquelas que tiveram maiores níveis de atividade física e baixo consumo de alimentos de risco $(p<0,01)$. Menores níveis de atividade física associados ao elevado consumo de alimentos protetores aumentaram a média do IMC em $0,68 \mathrm{~kg} / \mathrm{m}^{2}$, em comparação com maiores níveis de atividade física e alto consumo de alimentos protetores. A intervenção mostrou associação positiva com os parâmetros avaliados.

Obsidade; Antropometria; Exercício; Saúde da Mulher e aumento do nível de atividade física funciona positivamente na reversão da tendência de aumento da prevalência do excesso de peso e da obesidade, prevenindo suas conseqüências. Reconhece-se também, a necessária abordagem multiprofissional que requer esta estratégia, uma vez que mudanças de comportamento são complexas, principalmente ao se levar em conta questões alimentares, que se expressam em uma rede de significados sociais, culturais e emocionais. Além disso, essas estratégias devem ser implementadas de maneira continuada, com ênfase em atividades educativas, visando à conscientização dos envolvidos, e continuamente avaliada, para permitir adequações e adaptações que resultem de fato em mudanças positivas no estilo de vida.

\section{Colaboradores}

P. R. F. Costa participou da coleta, análise dos dados e redação do manuscrito. A. M. O. Assis e S. M. C. Pinheiro contribuíram na análise dos dados e redação do manuscrito. M. C. M. Silva, M. L. P. Santana e J. C. Dias colaboraram na coleta de dados e redação do manuscrito. N. S. Santos participou da redação do manuscrito.

\section{Agradecimentos}

À Secretaria Municipal de Saúde de Mutuípe, Bahia, Brasil, pela parceria. 


\section{Referências}

1. Organização Mundial da Saúde. Estratégia global em alimentação saudável, atividade física e saúde. Genebra: Organização Mundial da Saúde; 2004.

2. Organização Mundial da Saúde. Prevenção de doenças crônicas: um investimento vital. Brasília: Organização Mundial da Saúde; 2005.

3. Organização Pan-Americana da Saúde. Doenças crônico-degenerativas e obesidade: estratégia mundial sobre alimentação saudável, atividade física e saúde. Brasília: Organização Pan-Americana da Saúde; 2003.

4. Kain J, Vio F, Albala C. Obesity trends and determinant factors in Latin America. Cad Saúde Pública 2003; 19 Suppl 1:S77-86.

5. Instituto Brasileiro de Geografia e Estatística. Pesquisa de Orçamentos Familiares 2002-2003: análise da disponibilidade domiciliar de alimentos e do estado nutricional no Brasil. Rio de Janeiro: Instituto Brasileiro de Geografia e Estatística; 2004.

6. Ministério da Saúde. Obesidade. Brasília: Ministério da Saúde; 2006. (Cadernos de Atenção Básica, 12. Série A. Normas e Manuais Técnicos).

7. Monteiro CA, Conde WL, Popkin BM. Income-specific trends in obesity in Brazil: 1975-2003. Am J Public Health 2007; 97:1808-12.

8. Monteiro CA, D'A Benício MH, Conde WL, Popkin BM. Shifting obesity trends in Brazil. Eur J Clin Nutr 2000; 54:342-6.

9. Organización Mundial de La Salud, Food and Agriculture Organization. Dieta, nutrición y prevención de enfermedades crônicas. Geneva: Organización Mundial de la Salud; 2003.

10. World Health Organization. Obesity: preventing and managing the global epidemic. Report of a WHO Consultation Group on Obesity. Geneva: World Health Organization; 1997.

11. World Health Organization. The World Health Report 2002: reducing risks, promoting healthy life. Geneva: World Health Organization; 2002.

12. Masson CR, Dias-da-Costa JS, Olinto MTA, Meneghel S, Costa CC, Bairros F, et al. Prevalência de sedentarismo nas mulheres adultas da cidade de São Leopoldo, Rio Grande do Sul, Brasil. Cad Saúde Pública 2005; 21:1685-95.

13. Siqueira FV, Facchini LA, Piccini RX, Tomasi E, Thume E, Silveira DS, et al. Atividade física em adultos e idosos residentes em áreas de abrangência de unidades básicas de saúde de municípios das regiões Sul e Nordeste do Brasil. Cad Saúde Pública 2008; 24:39-54.

14. Haennel RG, Lemire F. Physical activity to prevent cardiovascular disease. How much is enough? Can Fam Physician 2002; 48:65-71.

15. World Health Organization. World health day 2002. Sedentary lifestyle: a global public health problem. http://www.who.int/world-health-day/ (acessado em 28/Dez/2007).

16. Ministério da Saúde. A vigilância, o controle e a prevenção das doenças crônicas não transmissíveis: DCNT no contexto do Sistema Único de Saúde. Brasília: Ministério da Saúde; 2005.

17. Behi R, Nolan M. Quasi experimental research designs. Br J Nurs 1996; 5:1079-81.
18. Kac G, Velásquez-Meléndez G, Coelho MASC. Fatores associados à obesidade abdominal em mulheres em idade reprodutiva. Rev Saúde Pública 2001; 35:46-51.

19. Barbosa PJB, Lessa I, Almeida Filho N, Magalhães LBNC, Araújo J. Criteria for central obesity in a Brazilian population: impact on the metabolic syndrome. Arq Bras Cardiol 2006; 87:407-14.

20. Lohman TG, Roche AF, Martorell R. Anthropometric standardization reference manual: Abridged edition. Champaign: Human Kinetics Books; 1988.

21. Organização Mundial da Saúde, Food and Agriculture Organization Organização das Nações Unidas. Necessidades de energia e proteína. São Paulo: Editora Roca; 1998.

22. World Health Organization. Physical status: the use and interpretation of anthropometry. Report of a WHO Expert Committee. Geneva: World Health Organization; 1995.

23. Monteiro RCA, Riether PTA, Burini RC. Efeito de um programa misto de intervenção nutricional e exercício físico sobre a composição corporal e os hábitos alimentares de mulheres obesas em climatério. Rev Nutr 2004; 17:479-89.

24. Haile RW, Hunt IF, Buckley J, Browdy BL, Murphy NJ, Alpers D. Identifying a limited number of foods important in supplying selected dietary nutrients. J Am Diet Assoc 1986:611-6.

25. Matsudo S, Araújo T, Matsudo V, Andrade D, Andrade E, Oliveira L, et al. Questionário Internacional de Atividade Física (IPAQ): estudo de validade e reprodutibilidade no Brasil. Rev Bras Ativ Fís Saúde 2001; 6:5-18.

26. Matsudo SM, Matsudo VR, Araújo T, Andrade D, Andrade E, Oliveira L, et al. Nível de atividade física da população do Estado de São Paulo: análise de acordo com o gênero, idade e nível sócio-econômico, distribuição geográfica e de conhecimento. Rev Bras Ciênc Mov 2002; 10:41-50.

27. Benedetti TRB, Antunes PC, Rodriguez-Añez CR, Mazo GZ, Petroski EL. Reprodutibilidade e validade do Questionário Internacional de Atividade Física (IPAQ) em homens idosos. Rev Bras Med Esporte 2007; 13:11-6.

28. Rabacow FM, Gomes MA, Marques P, Benedetti TRB. Questionários de medidas de atividade física em idosos. Rev Bras Cineantropom Desempenho Hum 2006; 8:99-106.

29. Rezende FAC, Rosado LEFPL, Ribeiro RCL, Vidigal FC, Vasques ACJ, Bonard IS, et al. Índice de massa corporal e circunferência abdominal: associação com fatores de risco cardiovascular. Arq Bras Cardiol 2006; 87:728-34.

30. Brown KH, Black RE, Becker S. Seasonal changes in nutritional status and the prevalence of malnutrition in a longitudinal study of young children in rural Bangladesh. Am J Clin Nutr 1982; 36:303-13.

31. Twisk JWR. Applied longitudinal data analysis for epidemiology: a practical guide. Cambridge: Cambridge University Press; 2003.

32. Medronho RA, Carvalho DM, Bloch KV, Luiz RR, Werneck GL. Epidemiologia. São Paulo: Editora Atheneu; 2006. 
33. Bo S, Ciccone G, Baldi C, Benini L, Dusio F, Forastiere G, et al. Effectiveness of a lifestyle intervention on metabolic syndrome: a randomized controlled trial. J Gen Intern Med 2007; 22:1695-703.

34. Jakicic JM, Clark K, Coleman E, Donnelly JE, Foreyt J, Melanson E, et al. American College of Sports Medicine position stand. Appropriate intervention strategies for weight loss and prevention of weight regain for adults. Med Sci Sports Exerc 2001; 33:2145-56.

35. Christ M, Iannello C, Iannello P, Grimm W. Effects of a weight reduction program with and without aerobic exercise in the metabolic syndrome. Int J Cardiol 2004; 97:115-22.

36. Radhika G, Sudha V, Sathya RM, Ganesan A, Mohan V. Association of fruit and vegetable intake with cardiovascular risk factors in urban south Indians. Br J Nutr 2008; 99:398-405.

37. Neumann AICP, Martins IS, Marcopito LF, Araujo EAC. Padrões alimentares associados a fatores de risco para doenças cardiovasculares entre residentes de um município brasileiro. Rev Panam Salud Pública 2007; 22:329-39.

38. Geraldo JM, Alfenas RCG, Alves RDM, Salles VF, Queiroz VMV, Bitencourt MCB. Intervenção nutricional sobre medidas antropométricas e glicemia de jejum de pacientes diabéticos. Rev Nutr 2008; 21:329-40.
39. V Diretrizes Brasileiras de Hipertensão Arterial. Arq Bras Cardiol. 2007; 89:e24-79.

40. Francischi RPP, Pereira LO, Freitas CS, Klopfer M, Santos RC, Vieira P, et al. Obesidade: atualização sobre sua etiologia, morbidade e tratamento. Rev Nutr 2000; 13:17-28.

41. Monteiro CA, Conde WL, Matsudo SM, Matsudo VR, Bonseñor IM, Lotufo PA. A descriptive epidemiology of leisure-time physical activity in Brazil, 1996-1997. Rev Panam Salud Pública 2003; 14 246-54.

42. Mondini L, Monteiro CA. Mudanças no padrão de alimentação da população urbana brasileira (1962-1988). Rev Saúde Pública 1994; 28:433-9.

43. Assis MAA, Nahas MV. Aspectos motivacionais em programas de mudança de comportamento alimentar. Rev Nutr 1999; 12:33-41.

44. Harris AD, McGregor JC, Perencevich EN, Furuno JP, Zhu J, Peterson DE, et al. The use and interpretation of quasi-experimental studies in medical informatics. J Am Med Inform Assoc 2006; 13:16-23.

45. Campbell DT. Reforms as experiments. Am Psychol 1969; 24:409-29.

Recebido em 21/Jan/2009

Versão final reapresentada em 14/Abr/2009

Aprovado em 27/Abr/2009 